\title{
On Polytopes Cut by Flats
}

\section{J. Kincses}

Bolyai Institute, Szeged University, Szeged, Aradi Vértanúk tere 1, H-6720 Hungary

\begin{abstract}
We prove that if we cut a $d$-polytope by a $k$-flat, then the complex of the uncut faces has the same simple-homotopy type as the boundary complex of a $(d-k)$-polytope. We also investigate the combinatorial properties of the complex of the uncut faces and, as a corollary, we answer negatively a question of Goodman and Pach.
\end{abstract}

\section{Introduction}

Bruggeser and Mani [1] proved that the boundary complex of a convex polytope is shellable. In the context of collapsibility this result yields that if we remove two facets of a convex $d$-polytope, then the complex of the remaining faces collapses to a subcomplex which is isomorphic to the boundary complex of a $(d-1)$-polytope. In the first part of this paper we investigate the generalization of the above result and we prove that if we cut a $d$-polytope by a $k$-flat, then the complex of the uncut faces has the same simple-homotopy type as the boundary complex of a convex $(d-k)$ polytope. For $k=d-1$ we can prove collapsibility. As an application of this result we give a topological proof of a weaker version of a theorem of Goodman and Pach [3]. In the second part of the paper we investigate the combinatorial structure of the complex of the uncut faces. We prove the counterintuitive fact that for the $d$-simplex $\Delta_{d}$ and for each $k(2 \leq k \leq d-2)$ there is a $k$-flat $K$ intersecting $\Delta_{d}$ in general position such that any $(d-k)$-flat intersecting $\Delta_{d}$ also meets a proper face of $\Delta_{d}$ which is cut by $K$. This result answers negatively a question of Goodman and Pach [3].

\section{The Topological Structure of the Complex of Uncut Faces}

By a polyhedral complex we understand a finite set of convex polytopes, all of which are contained in the same euclidean space $\mathbf{R}^{d}$ with the usual closedness and 
intersection properties (see [4]). By $|K|$ we denote the corresponding polyhedron, $|K|=\bigcup_{P \in K} P$. Let $K$ be a complex, and suppose that $F \in K$ is a proper face of exactly one polytope $P \in K$. Then the complex $K^{\prime}=K \backslash\{F, P\}$ is obtained from $K$ by an elementary collapse (and $K$ is obtained from $K^{\prime}$ by an elementary anticollapse). We say that the complex $K$ collapses to the subcomplex $L$ iff $L$ can be obtained from $K$ by a finite sequence of elementary collapses. We say that the complexes $K$ and $L$ have the same simple-homotopy type if a sequence of elementary collapses and elementary anticollapses can transform $K$ into $K^{\prime}$ (see [2]).

Let $P$ be a $d$-dimensional convex polytope and let $L$ be a $k$-dimensional flat which intersects $P$ in general position (that is, $L$ intersects the interior of $P$ but does not intersect any face of $P$ with dimension at most $d-k-1$ ). Denote by $P^{n}$ the complex of faces of $P$ which are not cut by $L$. Our main result is:

Theorem 2.1. There is a subcomplex $S$ of $P^{n}$ which is isomorphic with the boundary complex of $a(d-k)$-polytope and $|S|$ is a strong deformation retract of $\left|P^{n}\right|$. Moreover, the complexes $S$ and $P^{n}$ have the same simple-homotopy type.

For two reasons we discuss first the special case when $k=d-1$. On one hand, in this case we can prove a stronger statement, and, on the other hand, this is the key step in proving the general case.

Denote by $H^{u}$ and $H^{l}$ the closed half-spaces determined by the hyperplane $H$ and we say that $H^{u}\left(H^{l}\right)$ is the upper (lower) half-space of $H$. We call a face $F$ of $P$ an upper (lower) face of $P$ or say it is beyond (beneath) $H$ iff $F \subseteq H^{u}\left(F \subseteq H^{l}\right.$ ). The complex of uncut faces can be partitioned into two disjoint subcomplexes $P^{n}=$ $P^{u} \cup P^{l}$ where $P^{u}\left(P^{l}\right)$ is the set of upper (lower) faces.

Lemma 2.2. The union of the upper (lower) faces of $P$ are collapsible to a point.

Proof. By symmetry, it is sufficient to prove the statement for $P^{u}$ only. We prove by induction on the dimension of $P$. For $d=1$ it is obviously true. Assume that it holds for every $(d-1)$-dimensional polytope and let $P$ be a $d$-dimensional polytope and let $H$ be a cutting hyperplane. We may suppose, by changing the position of $H$ a little bit if necessary, that there is no hyperplane parallel to $H$ containing more than one vertex of $P$. Let $H_{1}$ be the supporting hyperplane of $P$ parallel to $H$ and $H_{1} \subseteq H^{u}$. Now we move $H$ toward $H_{1}$ keeping parallel to $H$. Let $H_{t}$ be the family of these hyperplanes, $0 \leq t \leq 1, H_{0}=H$. The complex $P_{t}^{u}$ of faces of $P$ which are beyond $H_{t}$ changes only when $H_{t}$ passes through a vertex of $P$. Let $x_{1}, x_{2}, \ldots, x_{k}$ be the upper vertices of $P$ and let $H_{i_{i}}$ be the hyerplane through $x_{i}, 0<t_{1}<\cdots<t_{k}$. $P_{t_{i}}^{u}$ differs from $P_{t_{i+1}}^{u}$ only in the complex $C_{x_{i}}$ of those faces of $P$ which are beyond $H_{t_{i}}$ and $x_{i}$ is a vertex of each of them. If $V_{x_{i}}$ denotes the vertex figure (see [4]) of $P$ at $x_{i}$, then the complex $V_{x_{i}}^{u}$ of those faces of $V_{x_{i}}$ which are beyond $H_{t_{i}}$, by the induction hypothesis, is collapsible to a point. However, $C_{x_{i}}$ is a cone over $B=$ $C_{x_{i}} \cap P_{t_{i+1}}^{u}$ and, under the central projection from $x_{i}, B$ is isomorphic with $V_{x_{i}}^{u}$ and so $B$ is also collapsible to a point. To sum up, we get that $P_{t_{i}}^{u}=P_{t_{i+1}}^{u} \cup C_{x_{i}}$, where $C_{x_{i}}$ is a cone over $B=C_{x_{i}} \cap P_{t_{i+1}}^{u}$ and $B$ is collapsible to a point. Nevertheless, it is a well-known fact (see [2]) that a cone collapses to its base if the base is collapsible 
to a point. This gives that $P_{t_{i}}^{u}$ collapses to $P_{t_{i+1}}^{u}$. Proceeding this way we finally have that $P_{t_{1}}^{u}=P^{u}$ collapses to $P_{t_{k}}^{u}=x_{k}$.

For the proof of Theorem 2.1 we need the following theorem which can be obtained as a combination of a result of Smale (see [8]) and Whitehead's theorem on the homotopy type of complexes.

Smale's Theorem. Let $K, L$ be complexes. If a map $f:|K| \rightarrow|L|$ is onto and for any point $x \in|L|$ the set $f^{-1}(x)$ is contractible to a point, then the map $f$ is a homotopy equivalence.

Proof of Theorem 2.1. We may suppose that the origin 0 is in $L \cap$ int $P$. Let $L^{\perp}$ be the orthogonal complement of $L$ and let $p_{L}: \mathbf{R}^{d} \rightarrow L^{\perp}$ be the orthogonal projection and $P^{\perp}=p_{L}(P)$. The shadow boundary of $P$ with respect to $L$ is defined as $p_{L}^{-1}\left(\mid \mathrm{bd} P^{\perp}\right) \cap P$, where bd $P^{\perp}$ denotes the boundary complex of $P^{\perp}$. Obviously, there is a subcomplex $S_{L}$ of $P^{n}$ such that $\left|S_{L}\right|$ is the shadow boundary. We may suppose, by changing the position of $L$ a little bit if necessary, that the shadow boundary of $P$ with respect to $L$ is sharp, that is, for any point $x \in$ bd $P \perp$ the set $p_{L}^{-1}(x) \cap P$ consists of exactly one point (note that, because $L$ cuts $P$ in general position, a sufficiently small change of $L$ does not change the set of uncut faces!). This gives that $p_{L}$ induces isomorphism between $S_{L}$ and bd $P^{\perp}$. Let $c: P^{\perp} \backslash 0 \rightarrow$ bd $P^{\perp} \mid$ be the central projection from the origin and consider the composite map $f=p_{L}^{-1} c p_{L}:\left|P^{n}\right| \rightarrow\left|S_{L}\right|$. Obviously, $f$ is continuous and it is the identity on $S_{L}$, that is, $f$ is a retraction of $P^{n}$ onto $S_{L}$. For an arbitrary point $x \in\left|S_{L}\right|$ we have that $f^{-1}(x)=\left|P^{n}\right| \cap L_{x}^{+}$where $L_{x}^{+}$is the half $(k+1)$-flat of the $(k+1)$-flat $L_{x}$ spanned by $L$ and $x$. The convex $(k+1)$-polytope $P \cap L_{x}$ is cut by the $k$-flat $L$ in general position and one of the components of the union of the uncut faces is exactly $\left|P^{n}\right| \cap L_{x}^{+}$. This implies, using Lemma 2.2, that $f^{-1}(x)$ is contractible to a point. Applying Smale's theorem, we have that $f$ is a homotopy equivalence. However, if a retraction is a homotopy equivalence, then it is a strong deformation retraction [9].

The second part of Theorem 2.1 is an easy consequence of the first part, by using the following result (see [2]):

If $K$ is a complex and $L$ is a subcomplex such that $L$ is a strong deformation retract of $K$ and the fundamental group of $K$ is either trivial or the group of integers, then $K$ and $L$ have the same simple-homotopy type.

This completes the proof of theorem.

We believe that in Theorem 2.1 the term simple-homotopy type can be replaced by collapsibility:

Question. Is is true that the complex $P^{n}$ collapses to the complex $S_{L}$ ?

For later use we determine the homotopy type of the union of the relative interior of the faces cut by $L$ (this union is denoted by $P^{c}$ ). 
Lemma 2.3. A continuous map $r: P^{c} \rightarrow L \cap P^{c}$ exists such that if $x$ is on a face of $P$, then $r(x)$ is on the same face of $P$ and $r(x)=x$ for $x \in P^{c} \cap L$, that is, $P \cap L$ is a strong deformation retract of $P^{c}$.

Proof. We call a subset of $P^{c}$ of the form $P^{c} \cap F \neq \varnothing$ an $i$-dimensional cell of $P^{c}$, where $F$ is an $i$-dimensional proper face of $P$. We define $r$ by induction on the dimension of the cells. If $P^{c} \cap F$ is a $(d-k)$-cell (there are no $(d-k-1)$-cells), then we define the map $r$ by sending each point of $P^{c} \cap F$ into the point $F \cap L$. Suppose that $r$ has been defined on each cell $P^{c} \cap F^{\prime}$ with dimension less than $j$ such that $r\left(P^{c} \cap F^{\prime}\right) \subseteq F^{\prime} \cap L, r(x)=x$ for $x \in F^{\prime} \cap L$ and let $P^{c} \cap F$ be a $j$-cell. Then $r$ is defined on the relative boundary of it and also on $L \cap F$ (identity) and $r$ agrees on the common parts of different cells. However, $\left(P^{c} \cap \operatorname{relbd} F\right) \cup$ $(L \cap F)$ is a closed subset of the cell and $r$ maps continuously this set into the $(j-d+k$ )-polytope $H \cap F$. By Tietze's theorem $r$ can be extended to the whole $j$-cell. For different $j$-cells these extended maps are equal on the common part, thus these maps can be fitted together to yield a map on the union of $j$-cells with the desired properties.

In the special case when the cutting flat is a hyperplane $H$, we can map the intersection $P \cap H$ into the upper (lower) part of the uncut faces.

Lemma 2.4. There are continuous maps

$$
p^{u}: H \cap P^{c} \rightarrow P^{u}, \quad p^{l}: H \cap P^{c} \rightarrow P^{l},
$$

such that $p^{u}$ and $p^{l}$ are homotopic to a constant and if $x$ is on a face of $P$, then $p^{u}(x)$, $p^{l}(x)$ are on the same face of $P$.

Proof. Consider the polytope $P \cap H^{u}$. Each $j$-face $F$ of $P \cap H$ is a face of a unique $\left(j+1\right.$ )-face $F^{\prime}$ of $P \cap H^{u}, F^{\prime} \nsubseteq H$ (since $H$ does not contain any vertices of $P$ ). For each $j$-face $(0 \leq j \leq d-1) F$ of $P \cap H$ let $\rho_{F}$ be the central projection in aff $F^{\prime}$ from a point which is sufficiently close to the centroid of $F$ and it is in $H^{l} \cap$ aff $F^{\prime} . \rho_{F}$ is a retraction from $F^{\prime}$ onto relbd $F^{\prime} \backslash$ relint $F$. These retractions can be fitted together to yield a retraction $\rho_{j}$ from the union of the $(j+1)$-faces of $P \cap H^{u}$ intersecting $H$. Then the map

$$
\rho_{0} \cdots \rho_{d-1} \rho_{d}: P \cap H^{u} \rightarrow P^{u}
$$

is a retraction and let $p^{u}$ be the restriction of it to (bd $\left.P\right) \cap H$. Then $p^{u}(x)$ and $x$ are on the same face and $p^{u}$ is homotopic to a constant. For the latter we use the fact that a continuous map from the $(d-1)$-sphere into an arbitrary space is homotopic to a constant if and only if it can be extended to the $d$-ball.

The map $p^{l}$ can be constructed in the same way as $p^{u}$.

As an application of the above results we prove a weaker version of a theorem of Goodman and Pach (see [3]). 
Corollary 2.5. If the hyperplane $H$ cuts the convex polytope $P \subseteq \mathbf{R}^{d}$ but does not contain any vertices of $P$, then for each point $x$ of $P$ there is a line passing through the point $x$ which meets the boundary of $P$ in only uncut faces.

Proof. Choose a point $x$ of $P$. We may suppose that $x$ is an interior point of $P$ because if it is a boundary point, then we can work on the face of $P$ containing $x$ in its relative interior. Introducing the map $c_{x}$ : bd $P \rightarrow$ bd $P$ which corresponds to a point $y$ of $\operatorname{bd} P$, the unique intersection point of bd $P$, and the line through $x$ and $y$ which is different from $y$, we must prove that $c_{x}\left(P^{l}\right) \cap P^{u} \neq \varnothing$ or, equivalently, $P^{l} \cap c_{x}\left(P^{u}\right) \neq \varnothing$. We may suppose that $x \in H^{u}$. In this case $c_{x}\left(P^{l}\right)$ is disjoint from $P^{l}$ and suppose, on the contrary, that $c_{x}\left(P^{l}\right)$ is disjoint from $P^{u}$. Then $c_{x}$ maps $P^{l}$ into $P^{c}$. We have, by Lemmas 2.3 and 2.4 , that the map

$$
r c_{x} p^{l}: P^{c} \cap H \rightarrow P^{c} \cap H
$$

is continuous and homotopic to a constant. However, $P^{c} \cap H$ is the boundary of the (d-1)-polytope $H \cap P$ and so $P^{c} \cap H$ is homeomorphic to the $(d-2)$-sphere. This gives that $r c_{x} p^{l}$ has a fixed point $x_{0}$. Let $F_{0}$ be the proper face of $P$ containing $c_{x} p^{l}\left(x_{0}\right)$. By the properties of the maps we have that $x_{0}$ and $p^{l}\left(x_{0}\right)$ are also on $F_{0}$ which contradicts that $x$ is an interior point of $P$.

Thus we proved the corollary.

\section{On the Combinatorial Structure of the Complex of Uncut Faces}

In this section we prove the counterintuitive fact that the union of the cut faces can be so "big" that it cannot be avoided by any $(d-k)$-flat intersecting the interior of the polytope. Let $\Delta_{d}$ be the $d$-dimensional simplex.

Theorem 3.1. If $d \geq 4$, then for each $k(2 \leq k \leq d-2)$ there is a $k$-flat $L$ intersecting $\Delta_{d}$ in general position such that any $(d-k)$-flat intersecting $\Delta_{d}$ also meets a proper face of $\Delta_{d}$ which is cut by $L$.

We prove this in two steps. The first one is the following lemma, which was proved by Prabhu and Perles (see [6]).

Lemma 3.2. For each $k(2 \leq k \leq d-2)$ there is a $k$-flat $L$ intersecting $\Delta_{d}$ in general position such that $L$ intersects the relative interior of every faces of $\Delta_{d}$ with dimension at least $d-[k / 2]([\cdot]$ means the integer part $)$.

Proof. Let $C$ be the $k$-dimensional cyclic polytope (see [4]) with $d+1$ vertices, the vertex set is $V=\left\{x_{1}, x_{2}, \ldots, x_{d+1}\right\}$. Let $\bar{V}=\left\{\bar{x}_{1}, \bar{x}_{2}, \ldots, \bar{x}_{d+1}\right\}$ be a Gale diagram (see p. $100[5]$ ) of the set $V$. Now we take $\bar{V}$ to be the image under an affine mapping $\Phi$ of the ordered set of vertices of $\Delta_{d}$. We state that the required flat is $L=$ $\operatorname{ker} \Phi=\Phi^{-1}\{0\}$. 
We use the Gale diagram relationship (see 3A1 on p. 100 of [5]):

If $\bar{X}$ is a Gale diagram of $X$, then $Y \subseteq X$ is a facial set if and only if $\bar{Y}=\left\{\bar{x}_{i} \in \bar{X} \mid\right.$ $\left.x_{i} \notin Y\right\} \subseteq \bar{X}$ is such that $0 \in \operatorname{relint} \operatorname{conv} \tilde{Y}$.

It is well known that the polytope $C$ is $[k / 2]$ neighborly, that is, every subset of its vertices with cardinality at most $[k / 2]$ spans a face of $C$. Using the Gale diagram relationship we have that for each subset $S$ of $\bar{V}$ with cardinality at least $d+1-$ $[k / 2]$ the relation $0 \in$ relint conv $S$ holds which means that the face of $\Delta_{d}$ corresponding to $S$ is cut by $L$ in its relative interior.

The polytope $C$ is simplicial, so, using again the Gale diagram relationship, we get that there is no nonempty subset $H$ of $\widetilde{V}$ with cardinality at most $d-k$ such that $0 \in$ relint conv $H$ which gives that $L$ meets $\Delta_{d}$ in general position.

Lemma 3.3. Let $k$ be an integer $2 \leq k \leq d-2$ and $k \neq 3$ or $d \neq 5$. If $a(d-k)$-flat $L$ intersects the interior of $\Delta_{d}$, then $L$ also intersects the relative interior of a proper face of $\Delta_{d}$ with dimension at least $d-[k / 2]$.

Proof. Obviously, we may suppose that $L$ contains the origin and the barycenter of $\Delta_{d}$ is the origin. Project $\Delta_{d}$ along the flat $L$ into the $k$-flat $L^{\perp}$ orthogonal to $L$. Denote the images of the vertices of $\Delta_{d}$ by $\bar{X}=\left\{\bar{x}_{1}, \bar{x}_{2}, \ldots, \bar{x}_{d+1}\right\}$. We have (see 2B4 on p. 97 of [5]) that there is a $(d-k)$-dimensional point set $X$ such that the Gale transform of $X$ is $\bar{X}$. Now for each vertex $v$ of conv $X$ let $S_{v}$ be the subset of $X$ consisting of points equal to $v$. Obviously, there is a vertex such that

$$
\left|S_{v}\right| \leq\left[\frac{d+1}{d-k+1}\right] .
$$

The set $S_{v}$ is clearly facial, so, using the Gale diagram relationship, it is enough to prove that

$$
\left[\frac{d+1}{d-k+1}\right] \leq\left[\frac{k}{2}\right] .
$$

Now let $d+1=l(d-k+1)+r(0 \leq r \leq d-k)$. Then $(*)$ can be reduced to

$$
l \leq \frac{k}{2}=\frac{l(d-k+1)+r-(d-k+1)}{2} .
$$

Simple calculation gives that it is enough to prove that

$$
1+\frac{2}{d-k-1} \leq l,
$$

which is true if $l \geq 3$ because $d-k-1 \geq 1$. If $l=1$, then $(*)$ is reduced to

$$
1 \leq\left[\frac{k}{2}\right] \text {, }
$$


which is certainly true since $k \geq 2$. If $l=2$, then $d+1=2(d-k+1)+r$, that is, $k=d-k+1+r$ and, since $d-k+1 \geq 3$, thus $k \geq 3$. This gives that

$$
2 \leq \frac{k}{2}=\frac{d-k+1+r}{2}
$$

except in the case when $k=3, r=0$, and $d=5$, which was excluded.

Proof of Theorem 3.1. Using Lemmas 3.2 and 3.3 we should only discuss the exceptional case $d=5$ and $k=3$.

Let $H=\{a, b, c,-a,-b,-c\}$ be the set of vertices of a regular hexagon centered at the origin such that $\operatorname{conv}\{a, b\}$ and $\operatorname{conv}\{b, c\}$ are edges of the hexagon. Now we take $H$ to be the image under an affine mapping $\Phi$ of the ordered set of the vertices of $\Delta_{5}$. Pick a point $x$ in the interior of the triangle conv\{a,b,0\} close enough to 0 . Let $L$ be the 3-flat $\Phi^{-1}\{x\}$ and take an arbitrary 2-flat $T$ intersecting the interior of $\Delta_{5}$.

If $T$ cuts the relative interior of a 4-face of $\Delta_{5}$, then we are ready because $L$ meets each 4-face of $\Delta_{5}$.

If however $T$ does not cut any 4-face of $\Delta_{5}$, then projecting $\Delta_{5}$ along $T$ into the three-dimensional space orthogonal to $T$, the image of the vertex set of $\Delta_{5}$ must be the following type (by a theorem of Steinitz, see Theorem 1.3 [7]):

$$
\left\{x_{1},-\lambda_{1} x_{1}, x_{2},-\lambda_{2} x_{2}, x_{3},-\lambda_{3} x_{3}\right\},
$$

where $x_{1}, x_{2}, x_{3}$ is a basis and the $\lambda_{i}$ 's are positive. In this case $T$ meets only three 3-faces of $\Delta_{5}$ such that the intersection of every two of them is an edge of $\Delta_{5}$. However, $L$ does not cut only three 3-faces of $\Delta_{5}$ and there are two out of these three faces which have a common 2 -face. This gives that $T$ cuts the relative interior of a 3-face of $\Delta_{5}$ which is cut by $L$ also.

Remark. Theorem 3.1 answers negatively the following question of Goodman and Pach [3]:

Let $L$ be a $k$-dimensional flat intersecting a d-polytope $P$ in general position (that is, $L$ does not intersect any faces of $P$ with dimension at most $d-k-1)$. Then, given any point $x \in P$, can we find a flat $T$ of dimension $d-k$ which passes through $x$ and meets the boundary of $P$ in only uncut faces?

\section{References}

1. H. Bruggeser and P. Mani, Shellable decompositions of cells and spheres, Math. Scand. 29 (1971), 197-205.

2. M. M. Cohen, A Course in Simple-Homotopy Type, Springer-Verlag, New York, 1973.

3. J. E. Goodman and J. Pach, Cell decomposition of polytopes by bending, Israel J. Math. 64 (1988), 129-138. 
4. B. Grünbaum, Convex Polytopes, Interscience, London, 1967.

5. P. McMullan, Transforms, diagrams and representations, in Contributions to Geometry (Proc. Geom. Symp., Siegen, 1978), Birkhäuser, Basel, 1979, pp. 92-130.

6. N. Prabhu, Properties of Convex Polytopes, Ph.D. Dissertation, New York University, 1991.

7. J. R. Reay, Generalizations of a Theorem of Carathéodory, Memoirs of the American Mathematical Society, vol. 54, American Mathematical Society, Providence, RI, 1965.

8. S. Smale, A Vietoris mapping theorem for homotopy, Proc. Amer. Math Soc. 8 (1957), 604-610.

9. E. H. Spanier, Algebraic Topology, McGraw-Hill, New York, 1966.

Received February 27, 1991, and in revised form April 13, 1994, and March 29, 1995. 